\title{
Primary medical therapy and breast conservation treatment: the medical oncology perspective
}

\author{
Nan Soon Wong \\ Oncocare Cancer Centre, Gleneagles Medical Centre, Singapore \\ Correspondence to: Dr. Nan Soon Wong. Oncocare Cancer Centre, Gleneagles Medical Centre, Singapore. Email: ns_wong@oncocare.sg.
}

\begin{abstract}
Primary systemic therapy (PST) is a widely adopted strategy for increasing operability and breast conservation rates. Although first generation PST trials failed to demonstrate improvements in disease free and overall survival compared to adjuvant systemic therapy (AST), they did demonstrate a strong association between attainment of pathologic complete response (pCR) and improved survival outcomes, leading to the widespread adoption of pCR as the primary endpoint in subsequent PST trials. First generation trials also showed that preoperative PST can improve breast conservation rates and downstage the axilla. Although individual trials did not demonstrate statistically significant increase in local recurrence with PST when compared to AST, a recent meta-analysis did note an increased in such risk, mainly driven by trials in which surgery was omitted in patients with good response to PST. Successive generations of PST clinical trials have since explored the activity of taxanes, optimization of anthracycline and taxane dose and schedules, incorporation of single and dual anti-HER2 therapy in HER2 overexpressing breast cancer, the use of platinums in triple negative breast cancer, and the role of endocrine therapy in hormone receptor positive breast cancer. While these PST trials have generally found increased pCR rates with the introduction of modern chemotherapy regimens and targeted therapies, they have not consistently demonstrated further improvements in breast conservation rates compared to first generation regimens. The reasons for this are complex and may lie beyond differences in anti-tumour activity between different systemic regimens but rather in other potential confounding factors such as tumour to breast volume ratio, tumour location, multicentricity as well as patient or surgeon preference.
\end{abstract}

Keywords: Primary systemic therapy (PST); pathologic complete response (pCR); breast conserving surgery; local recurrence

Submitted Jul 29, 2018. Accepted for publication Oct 08, 2018.

doi: $10.21037 /$ gs.2018.10.02

View this article at: http://dx.doi.org/10.21037/gs.2018.10.02

\section{Introduction}

Primary systemic therapy (PST) refers to the use of preoperative chemotherapy, endocrine therapy and/ or targeted therapy in the treatment of localized breast cancer. Other commonly used terms include preoperative systemic therapy, neoadjuvant therapy, induction therapy or downstaging therapy, all of which are used interchangeably in clinical practice (1).

First generation PST trials sought to determine if chemotherapy administered prior to surgery could increase the rate of breast conserving surgery, reduce the incidence of positive axillary nodes, as well as improve disease free and overall survival compared to the same chemotherapy administered after surgery (2-10). While these trials failed to demonstrate disease free or overall survival benefit with PST compared to adjuvant systemic therapy (AST) $(11,12)$, rates of breast conservation were increased $(2,6,12)$. In addition, the observation that patients who achieved pathologic complete response (pCR) after PST had improved long term survival outcomes compared to those with residual disease $(3,4,6)$ has led to the adoption of pCR as the primary endpoint in the majority of subsequent PST trials. 
Although successive generations of PST trials have demonstrated improvements in pCR rates with the optimal use of anthracyclines, taxanes and targeted agents, the corresponding improvement in breast conservation rates has been inconsistent across trials.

This article will review the evolution of PST, the impact of various therapeutic agents and schedules on pCR, breast conservation rates and risk of local recurrence.

\section{History of PST}

The biological basis for PST can be traced to preclinical models in the 1960s which showed that surgical removal of a primary tumour was associated with increased tumour growth at metastatic sites compared to controls in which primary tumours remained intact (13). Subsequent animal experiments showed that maximum suppression of tumour proliferation at metastatic sites could be achieved with chemotherapy given prior to surgical removal of the primary tumour compared to administration after surgery, raising hopes that PST could potentially improve patient survival compared to AST (14).

In the 1970s and 1980s, PST either alone or sequentially with radiotherapy was shown to result in high clinical response rates ranging from $70-90 \%(15,16)$, establishing its role as a standard treatment to achieve operability in locally advanced breast cancer.

In the 1990s, the indication for PST was expanded to include operable early breast cancers larger than $3-5 \mathrm{~cm}$ with the aim of achieving breast conservation. This was based on the observed high rate of clinical response and successful conversion from mastectomy to breast conserving surgery ranging from $80-90 \%$ in small single arm studies $(17,18)$. A further evolution has been the application of PST to reduce the extent of surgery with the aim of improving cosmesis in existing candidates for breast conservation (19).

More recently, PST can now allow a subset of patients with clinically positive axillary nodes to be spared the morbidity of axillary dissection (20). About $40-45 \%$ of patients are down-staged from clinically node positive disease to ypN0 after PST $(2,21,22)$, and sentinel lymph node biopsy has been found to be feasible in this scenario in carefully selected patients $(21,22)$.

In addition to the surgical benefit of PST in terms of downstaging of disease, it has been recognised since the 1980s that PST offers a unique opportunity to measure tumour response as a test of real time in-vivo sensitivity to various systemic therapies (23). From a research viewpoint, this allows for the design of correlative studies for the development of predictive biomarkers. Clinically, this permits discontinuation of ineffective therapy. More recently, the use of non-cross resistant adjuvant therapy for patients with residual disease despite optimal PST has been shown to improve disease free and overall survival in HER2 negative early breast cancer (24).

A key observation across virtually all randomized trials of PST is that patients who achieve complete pCR have significantly improved disease free and overall survival compared to patients who do not achieve pCR, especially in triple negative breast cancer, HER2 positive breast cancer and high grade ER positive breast cancer (3-5,25-28). This has led to the adoption of pCR as the primary endpoint in comparative studies evaluating the efficacy of various neoadjuvant chemotherapy regimens. Indeed, the 2014 US Food and Drug Administration guidance for industry on the use of pCR as an endpoint for accelerated approval of neoadjuvant therapeutic agents in high risk early stage breast cancer accepts that a novel agent that produces a marked absolute increase in pCR rate compared with standard therapy may be reasonably likely to result in long-term improvements in event free or overall survival (FDA) (29). This enables comparative trials of therapeutic agents with much fewer patient numbers and with determination of outcomes in a much shorter time frame measured in months compared to adjuvant studies which typically require 5 years of follow-up for definitive conclusions.

Definition of pCR has historically varied across different clinical trials but uniformity has been achieved in recent years. Eradication of invasive disease in the breast and lymph nodes (ypT0/isN0) as well as eradication of all disease including in-situ carcinoma (ypT0N0) are both better associated with event free survival (EFS) and overall survival (OS) than eradication of disease from breast alone (ypT0). Association with EFS and OS are similar for both ypT0N0 and ypT0/isN0, the latter being the most widely adopted definition of pCR $(27,28)$.

\section{First generation PST studies}

Large randomized clinical trials which compared modern day polychemotherapy regimens in the PST setting to the adjuvant setting are summarized in Table 1 .

These studies reported high clinical response rates between $49 \%$ and $82 \%(2-10)$. The pCR rates ranged from $3.7 \%$ to $20 \%$ and absence of estrogen receptor expression was found to be significantly associated with 


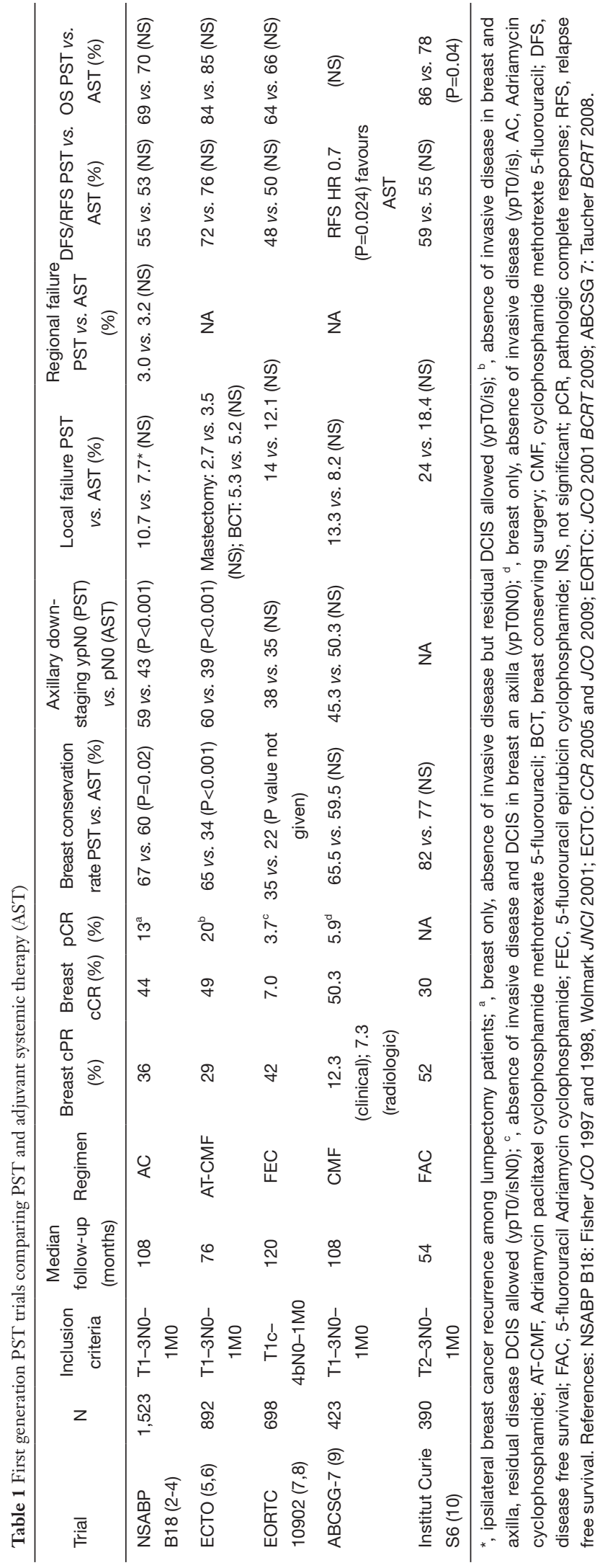

the increased probability of pCR (6). Axillary downstaging occurred in approximately $20 \%$ of patients in the NSABP $\mathrm{B} 18$ and ECTO trials, and breast conserving surgery was significantly increased in the PST arm of both trials $(67 \%$ vs. $60 \%, \mathrm{P}=0.02$ and $65 \%$ vs. $34 \%, \mathrm{P}<0.001$, respectively) (2-6).

Within individual trials, local and regional recurrence rates are similar for PST compared to AST. In the subgroup of patients who became eligible for breast conserving surgery as a result of tumour downstaging from PST, results are conflicting. In the NSABP B18 trial comparing pre $v$ s. postoperative doxorubicin cyclophosphamide (AC) documented a higher rate of ipsilateral breast cancer recurrence among patients who were converted from planned mastectomy to lumpectomy after PST compared to those who proceeded to lumpectomy as planned followed by AST (15.6\% vs. 9.9\%, $\mathrm{P}=0.04)$. This higher recurrence risk can be attributed partly to differences in the age distribution between the 2 groups (recurrence rate $\mathrm{P}$ value 0.14 after adjusting for age differences) (4). This contrasts with the EORTC 10902 study $(7,8)$ which showed no differences in locoregional recurrence rates between patients who underwent lumpectomy in the postoperative chemotherapy group, patients who converted from mastectomy to lumpectomy as a result of PST and also patients who were eligible for lumpectomy prior to chemotherapy (overall $\mathrm{P}$ value 0.97 ).

In the Early Breast Cancer Trialists Collaborative Group metanalysis of individual patient data from trials comparing PST versus AST, an increase in the rate of local recurrence was noted in patients who received PST (12). The 15 year local recurrence was $21.4 \%$ for PST $v s .15 .9 \%$ for adjuvant chemotherapy [rate ratio $(\mathrm{RR})=1.37 ; 95 \%$ CI: 1.17 1.61; $\mathrm{P}=0.0001]$. The absolute increase in 10 -year local recurrence with NACT was largest in the two trials $(10,30)$ in which, after PST, many women did not have breast surgery (33.7\% for PST vs. 20.4\% for AST; RR $=1.62 ; 95 \%$ CI: $1.20-2.19 ; \mathrm{P}=0.002)$. Excluding these 2 studies, the absolute increase in 10 year local recurrence was smaller but still statistically significant (difference 3.2\%; 95\% CI: $0.6-5.8 ; 15.1 \%$ vs. $11.9 \%$; RR $=1.28$; $95 \%$ CI: $1.06-1.55$; $\mathrm{P}=0.01$ ). The rate ratio for local recurrence comparing PST versus AST was higher in women who were planned to have mastectomy (1.66; 95\% CI: 1.24-2.21) compared to women who were planned to undergo breast conserving surgery (1.14; 95\% CI: 0.86-1.52), suggesting that the increase in local recurrence could potentially be attributed to the use of breast conserving surgery among patient initially allocated 
to mastectomy but converted to breast conserving surgery after PST.

Nonetheless, these trials provide reassurance that the efficacy of chemotherapy regimens in improving disease free and overall survival when administered in the adjuvant setting is not compromised when the same regimen is administered preoperatively. An outlier to this is ABCSG-07 (9), which compared 3 cycles of preoperative versus postoperative cyclophosphamide methotrexate 5-fluorouracil (CMF) and found inferior recurrence free survival in patients randomized to the PST. Possible explanations for this include a chance finding, given that no formal statistical plan for sample size calculations were provided and the trial accrual took 9 years to complete; as well as the low activity of preoperative CMF given for only 3 cycles.

\section{Second generation PST trials}

Second generation PST trials evaluated the role of taxanes (either paclitaxel or docetaxel) and the optimization of chemotherapy intensity and schedules and are summarized in Tables 2,3.

The Aberdeen (32-34) and NSABP B27 trials $(25,31)$ studied the addition of docetaxel sequentially after anthracycline while the Institute Curie (35) and AngloCeltic Cooperative Oncology Group (36) used a concurrent anthracycline taxane strategy.

In the NSABP B27 trial, compared to doxorubicin cyclophosphamide (AC) alone, preoperative AC followed by docetaxel (D) significantly increased the clinical response rate $(40.1 \%$ vs. $63.6 \% ; \mathrm{P}<0.001)$, the overall clinical response rate $(85.5 \%$ vs. $90.7 \% ; \mathrm{P}<0.001)$, the proportion of patients with negative axillary nodes $(50.8 \%$ vs. $58.2 \%$; $\mathrm{P}<0.001)$ and $\mathrm{pCR}$ rate $(13.7 \%$ vs. $26.1 \% ; \mathrm{P}<0.001)$. However, the rate of breast conservation was not increased (61.6\% vs. 63.7\%; $\mathrm{P}=0.33$ ). This was postulated to be due to only a modest increase in clinical objective response from $85.7 \%$ to $90.7 \%$ with the addition of preoperative docetaxel. In addition, the reasons for performing mastectomy versus breast conserving surgery was not formally collected in the trial and could have been due to reasons other than surgical factors. There was an association between clinical complete response (cCR) and breast conserving surgery, in that more patients with cCR underwent lumpectomies $(70.2 \%)$ than those without cCR (55.8\%). This was also the case for pCR (71.4\% lumpectomy) versus non pCR (60.3\% lumpectomy).

With long term follow-up, the incidence of local

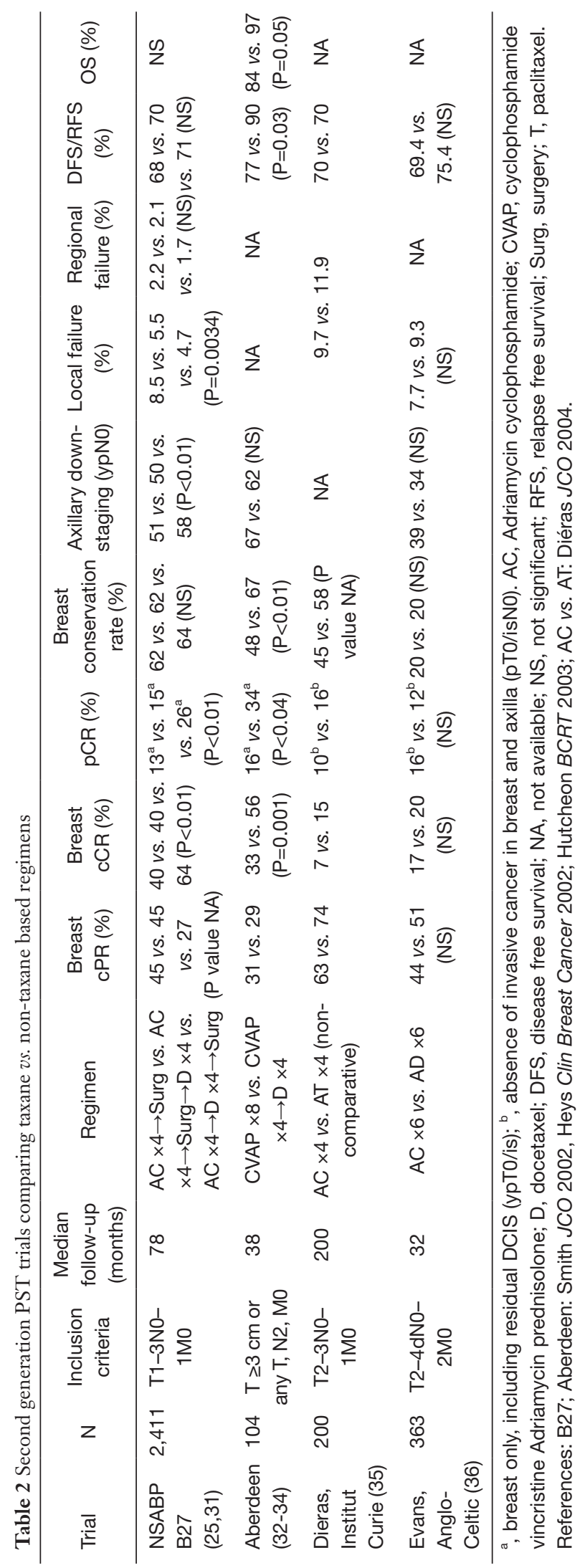




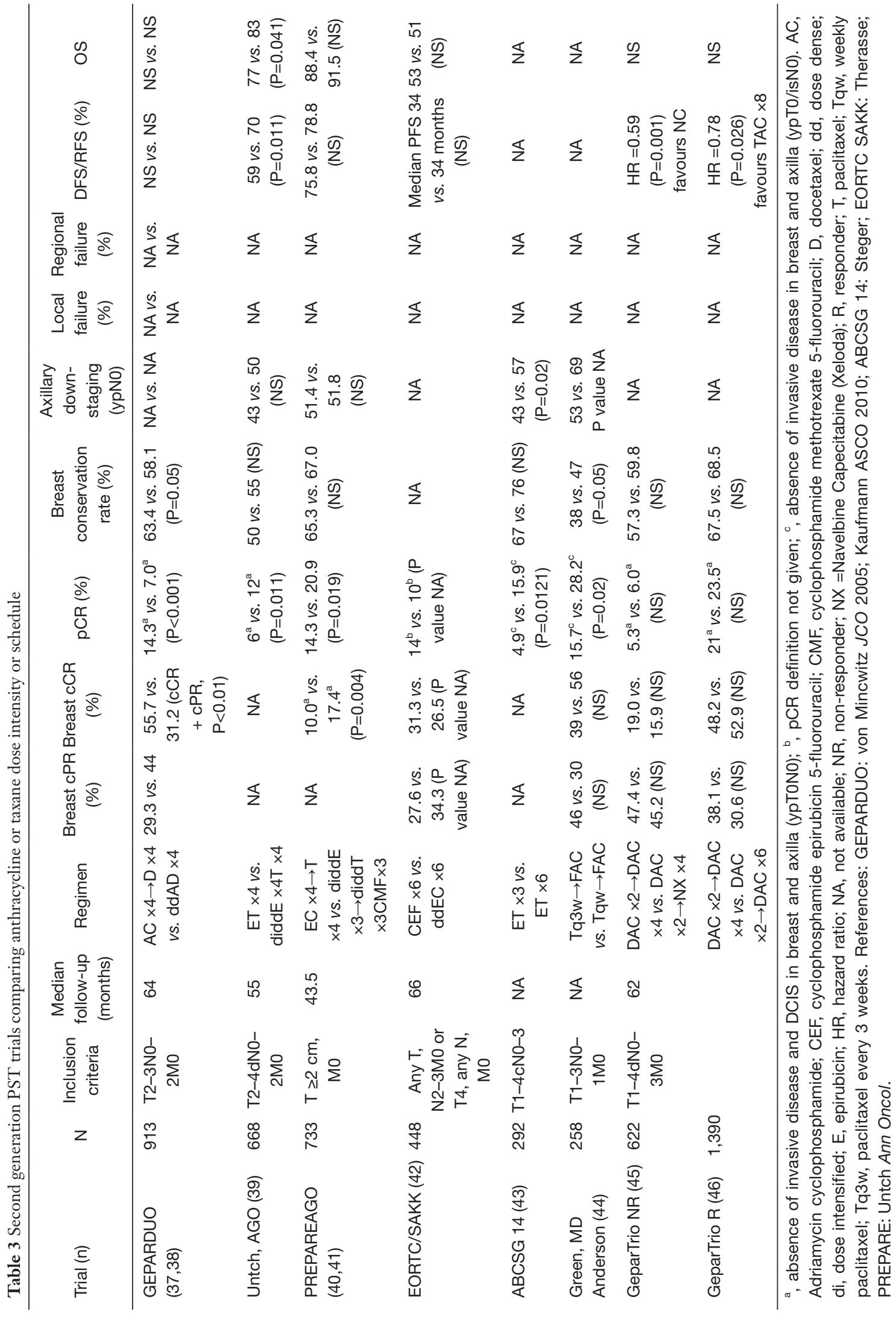


recurrence was significantly reduced with preoperative AC followed by D (4.7\%) or preoperative AC followed by adjuvant $\mathrm{D}(5.5 \%)$ compared to preoperative $\mathrm{AC}$ alone (8.5\%); $\mathrm{P}=0.0034$.

Disappointingly, the addition of either pre- or postoperative docetaxel did not improve DFS or OS. The primary reason for this has been postulated to be due to the anticipated 2-3\% improvement in DFS with a doubling of pCR rate from $13 \%$ to $26 \%$, a difference which would require a much larger trial to detect.

The Aberdeen trial (32-34) is a much smaller clinical trial $(n=104)$ which randomized patients with clinical partial or complete response after 4 cycles of anthracycline based therapy (CVAP) to continuation of the same regimen for 4 additional cycles or a switch to 4 cycles of docetaxel. In contrast to NSABP B27, switching to docetaxel increased the rate of breast conserving surgery as well as disease free and overall survival. Because of the small size of the Aberdeen trial, these findings will need to be confirmed in a larger study.

While NSABP B-27 and Aberdeen trials established a benefit from adding sequential taxanes to preoperative anthracyclines, the Anglo-Celtic Cooperative Oncology Group trial showed no benefit of concurrent doxorubicin docetaxel (AD) for 6 cycles compared to AC for 6 cycles in terms of clinical response rate, $\mathrm{pCR}$ rate, locoregional recurrence rate and long term survival outcomes (36). In a similarly designed Institut Curie trial comparing doxorubicin paclitaxel (AT) for 4 cycles to AC for 4 cycles, there was a numerical improvement in clinical objective response, pCR rate and breast conservation rate but not disease free survival. As this was a small parallel group non comparative trial, definitive conclusions cannot be drawn (35).

Preoperative sequential AC followed by D was formally compared to concurrent $\mathrm{AD}$ in the GeparDuo trial $(37,38)$, which showed an improvement in clinical response rate (85.0\% vs. $75.2 \% ; \mathrm{P}=0.001)$, pCR rate $(14.3 \%$ vs. $7.0 \%$; $\mathrm{P}<0.001)$ and successful breast conservation rate $(63.4 \%$ vs. $58.1 \% ; \mathrm{P}=0.05)$ favouring the sequential approach. Taken together with results of the Anglo-Celtic trial, the sequential administration of optimal doses of AC followed by $\mathrm{D}$ results in improved $\mathrm{pCR}$ compared to the combination of $\mathrm{AD}$ which results in reduced dose intensities of $\mathrm{A}$ and $\mathrm{D}$.

Optimal scheduling of paclitaxel was studied in an MD Anderson trial which compared paclitaxel every 3 weeks for 4 cycles followed by 5 -fluorouracil Adriamycin cyclophosphamide (FAC) for 4 cycles to weekly paclitaxel for 12 weeks followed by the same FAC for 4 cycles (44).
Clinical response rate was similar but pCR was increased with weekly paclitaxel (28.2\% vs. $15.7 \%$; $\mathrm{P}=0.02)$. Breast conservation rate was also increased ( $47 \%$ vs. $38 \% ; \mathrm{P}=0.05)$. In addition, there was a numerical increase in axillary ypN0 (69\% vs. 53\%, $\mathrm{P}$ value not provided).

A number of second generation PST trials have examined the impact of dose intensification and dose density on pCR rates and disease free survival.

The EORTC/NCIC/SAKK trial compared cyclophosphamide epirubicin 5-fluorouracil (CEF) every 4 weeks for 6 cycles versus epirubicin cyclophosphamide every 2 weeks for 6 cycles to achieve higher dose density but the same cumulative anthracycline dose (42). This study showed that increasing epirubicin dose intensity beyond $\mathrm{CEF}$ does not improve clinical response, $\mathrm{pCR}$ rate or disease free or overall survival.

AGO-1 trial (39) compared conventionally dosed combination epirubicin paclitaxel every 3 weeks for 4 cycles to dose dense, dose intensified sequential epirubicin for 4 cycles followed by paclitaxel for 4 cycles. All patients received 3 cycles of adjuvant cyclophosphamide methotrexate 5-fluorouracil (CMF) after surgery. pCR rate was improved $(12 \%$ vs. $6 \% ; \mathrm{P}=0.011)$ in the dose intensified dose dense arm which translated into an improvement in DFS (70\% vs. 59\%; $\mathrm{P}=0.011)$ and overall survival $(83 \%$ vs. $77 \% ; \mathrm{P}=0.041)$. There was no impact on breast conservation rates and axillary downstaging.

Contrasting with the results of AGO-1, the PREPARE trial $(40,41)$ compared conventional sequential epirubicin for 4 cycles followed by paclitaxel for 4 cycles $(\mathrm{Ex} 4 \rightarrow \mathrm{Tx} 4)$ with dose intensified dose dense sequential epirubicin for 3 cycles, paclitaxel for 3 cycles then CMF for 3 cycles $($ dddiEx3 $\rightarrow$ dddiTx3 $\rightarrow$ CMFx3). pCR rate was increased but this did not translate into a survival benefit. Breast conservation and axillary downstaging rates were not improved. The difference in survival outcomes between AGO-1 and PREPARE may have been contributed by the use of darbepoetin alpha in the latter study, which had a negative impact on overall survival but not on pCR rate.

Overall, both AGO-1 and PREPARE provide supporting evidence for dose dense and sequential rather than concurrent anthracycline taxane approach in the neoadjuvant setting.

In terms of duration of therapy, ABCDG 14 (43) compared 3 vs. 6 cycles of preoperative epirubicin paclitaxel (ET). Extending treatment to 6 cycles resulted in increased pCR rate $(15.9 \%$ vs. $4.9 \% ; \mathrm{P}=0.0121)$, breast conserving surgery rate $(47 \%$ vs. $38 \%, \mathrm{P}=0.05)$ and axillary ypN0 rate 
(69\% vs. 53\%, $\mathrm{P}$ value not provided).

The concept of response guided therapy (switching to a potentially non cross-resistant regimen in patients who do not respond to initial PST and intensifying therapy in responding patients) was tested in the GeparTrio study (45-47). Patients with no clinical response after 2 cycles of neoadjuvant docetaxel Adriamycin cyclophosphamide (TAC) were randomized to either continuation of TAC for 4 cycles or switch to vinorelbine capecitabine for 4 cycles (NX). Switching to NX did not increase the rate of clinical response, $\mathrm{pCR}$ or breast conservation. For patients with initial partial or complete clinical response to 2 cycles of TAC, 6 further cycles of TAC versus 4 cycles of TAC improved clinical response rate but not $\mathrm{pCR}$ or breast conservation. In an exploratory analysis, switching to NX in patients with early non response to TAC and extending TAC from 6 to 8 cycles in responding patients was associated with improvement in disease free survival (47).

\section{Third generation PST trials involving specific breast cancer subtypes/targeted therapy}

\section{Her2 positive breast cancer}

HER2 gene amplification is found in 20-25\% of breast cancers, leading to HER protein overexpression and development of an aggressive clinical course with shortened disease free and overall survival (48). PST trials involving HER2 positive breast cancer are summarized in Table 4.

The MD Anderson $(51,52)$ and NOAH $(49,50)$ trials randomized patients with HER2 positive breast cancer to receive anthracycline and taxane containing PST alone or with trastuzumab, a humanized anti-HER2 monoclonal antibody. Both studies reported high clinical response rates with chemotherapy trastuzumab combination (87-96\%). pCR rates were higher with the addition of trastuzumab (MD Anderson trial 65.2\% vs. 26\%, $\mathrm{P}=0.016$ and $\mathrm{NOAH}$ Trial $38 \%$ vs. $19 \% ; \mathrm{P}=0.001$ ) as was disease free survival (MD Anderson $100 \%$ vs. $85.3 \% ; \mathrm{P}=0.041$ and $\mathrm{NOAH}$ trial $65 \%$ vs. $47 \% ; \mathrm{P}=0.012$ ). Interestingly, despite significantly increased cCR and pCR rates with chemotherapy plus trastuzumab, breast conserving surgery rate was not increased in the MD Anderson trial (and not reported in the NOAH trial). This was attributed to patient preference and persistent abnormalities on imaging despite cCR on physical examination.

The activity of chemotherapy plus trastuzumab has been further confirmed in the GeparQuattro (53) and TECHNO (54) trials, both of which demonstrated high clinical response rates $(81.3 \%$ and $83 \%$, respectively), pCR rates $(40 \%$ and $38.7 \%$, respectively), breast conservation rates $(63.1 \%$ and $65 \%$, respectively) and rates of ypN0 (70\% and $70 \%$, respectively). In the TECHNO trials, tumours which were both estrogen and progesterone receptor negative had higher $\mathrm{pCR}$ rate than those which were estrogen or progesterone receptor positive (43.5\% vs. 23.4\%; $\mathrm{P}<0.001)$.

A large number of trials (55-61) have evaluated the role of lapatinib, an oral small molecule tyrosine kinase inhibitor of both epidermal growth factor receptor and HER2. These studies compared preoperative chemotherapy in combination with either lapatinib or trastuzumab or dual anti-HER2 blockade using the combination of trastuzumab and lapatinib. In general, these studies showed that pCR rates are lower with chemotherapy plus lapatinib compared with chemotherapy plus trastuzumab, reaching the level of statistical significance in the GeparQuinto trial (55). The combination of chemotherapy plus dual anti-HER2 blockade using trastuzumab and lapatinib showed clinical response rates between $80.2-90.8 \%$ and pCR rates between 46.7-60.2\%. In both the NEOALTTO (57) and CALGB 40601 (59) trials, pCR rates were higher in hormone receptor negative tumours compared to hormone receptor positive tumours. Despite higher rates of $\mathrm{pCR}$, there was no statistical increase in the rates of breast conserving surgery compared to chemotherapy plus lapatinib or chemotherapy plus trastuzumab. For example, in the NEOALTTO trial, pCR rate and ypN0 rate were increased for chemotherapy plus dual anti-HER2 therapy versus chemotherapy plus trastuzumab (46.8\% vs. $27.7 \% ; \mathrm{P}=0.0007$ and $73 \%$ vs. $58.6 \% ; \mathrm{P}=0.0115$, respectively), but the rate of breast conserving surgery was not different $(41.4 \%$ vs. $38.9 \%$; $\mathrm{P}$ value not significant). The impact of PST on eligibility and frequency of breast conserving surgery in HER2 positive breast cancer was explored in the CALGB 40601 surgical companion study (60). 59\% of patients in this trial were deemed to be ineligible for breast conserving surgery. PST was able to convert $43 \%$ of these patients from ineligible to eligible, increasing the overall proportion of patients deemed eligible for breast conserving surgery from $41 \%$ to $64 \%$. Despite this increase in the proportion of eligible patients, only $40 \%$ of patients in the trial eventually underwent breast conserving surgery. While this could have been due to patient and surgeon choice or preference, $44 \%$ of patients deemed ineligible for breast conserving surgery were actually found to have pCR at the time of surgery, suggesting the lack of optimal use of radiologic assessment 


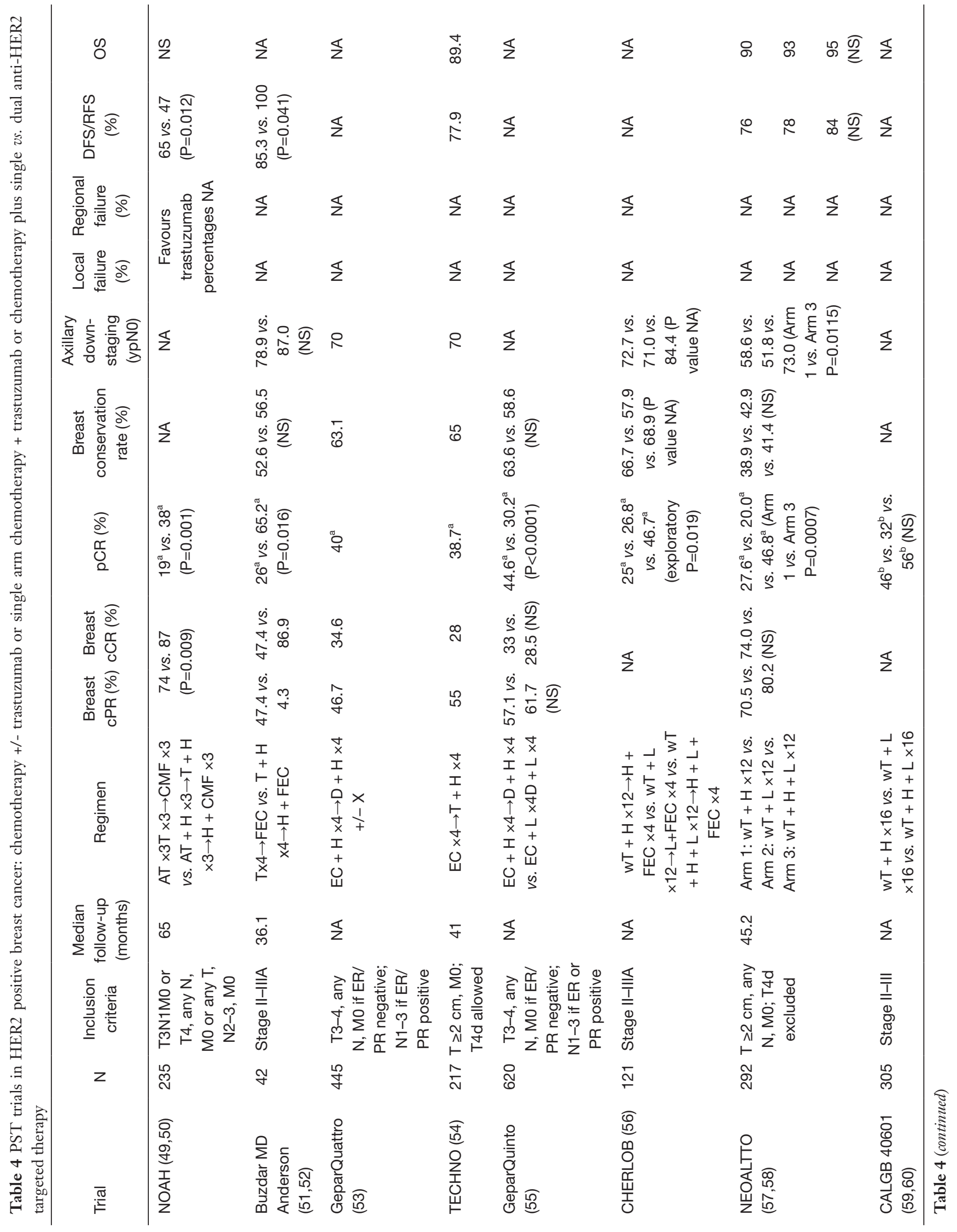




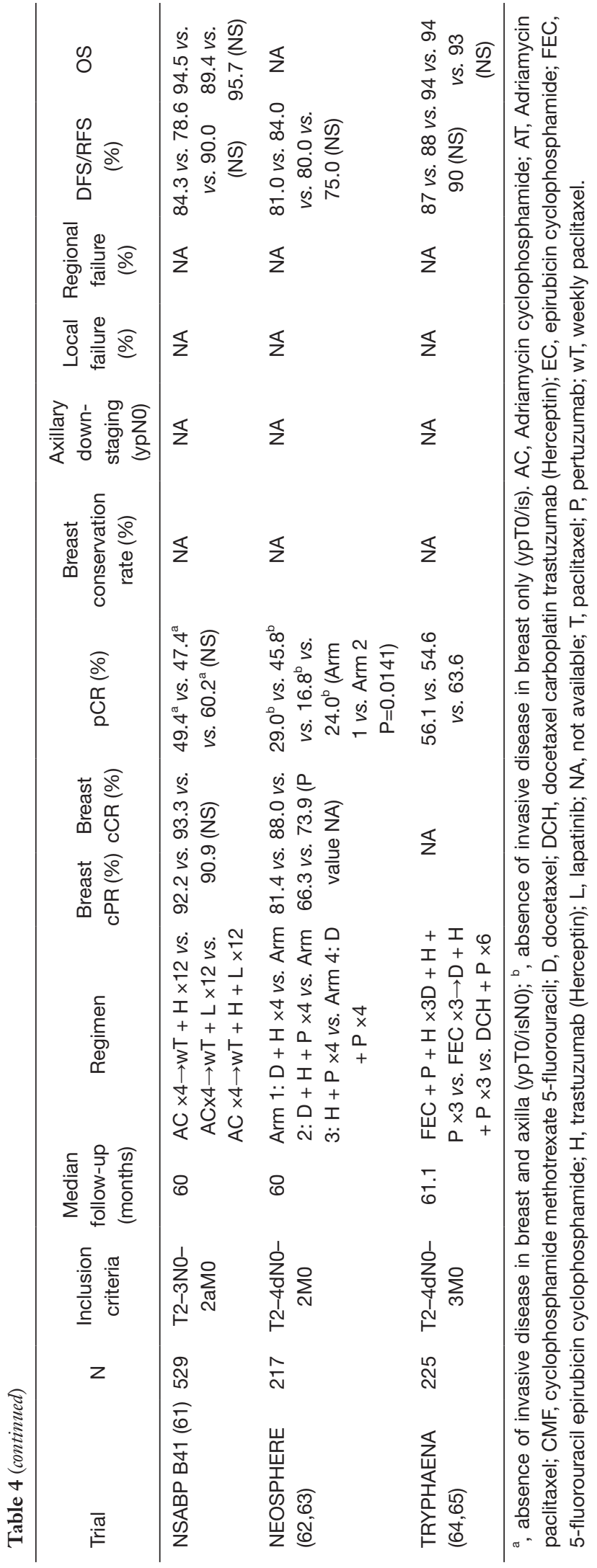

to determine suitability for breast conservation.

Neoadjuvant dual anti-HER2 blockade has also been explored using trastuzumab plus pertuzumab, a monoclonal antibody which binds to domain II of the HER2 receptor. In the NEOSPHERE trial $(62,63)$, pCR rate for the combination of docetaxel trastuzumab pertuzumab was $45.8 \%$ versus $29.0 \%$ for docetaxel trastuzumab $(\mathrm{P}=0.0141)$. Disease free survival was numerically higher in the docetaxel plus dual anti-HER2 arm but did not reach statistical significance given the small sample size. In the TRYPHAENA trial $(64,65)$, pCR rates were similar with FEC plus trastuzumab pertuzumab followed by docetaxel plus trastuzumab pertuzumab (56.1\%) vs. FEC alone followed by docetaxel plus trastuzumab pertuzumab (54.6\%) $v s$. a non-anthracycline containing combination of docetaxel carboplatin concurrent with trastuzumab and pertuzumab (63.6\%).

\section{Triple negative breast cancer}

Approximately $10-20 \%$ of breast cancers are hormone receptor and HER2 negative and are referred to as triple negative breast cancers. They tend to be clinically aggressive and share similar morphologic and molecular features as basal like cancers as defined by gene expression profiling (66). Due to the morphologic and immunohistochemical similarity between sporadic triple negative breast cancer, basal like breast cancer as well as BRCA1 mutated breast cancer, it has been postulated that sporadic triple negative breast cancer may harbour defects in homologous recombination and hence may be susceptible to DNA cross linking compounds such as platinums (67).

This hypothesis has been tested in a number of trials comparing anthracycline and taxane based PST with or without carboplatin (68-74) (Table 5). In these studies, pCR in the carboplatin plus chemotherapy ranged between $45.9 \%$ and $54 \%$, representing an increase of $10.5-25 \%$ compared to the chemotherapy alone arms. In the GeparSixto trial $(68,69)$, this translated into a disease free survival benefit with the addition of carboplatin $(85.8 \%$ vs. $76.1 \%, \mathrm{P}=0.035)$. In contrast, disease free survival was numerically higher in the carboplatin arm of CALGB $40603(76.5 \%$ vs. $71 \%)$ but this did not reach statistical significance (70-72). The reasons for this discordance is unclear but could be due to the small sample size of both studies, differing proportion of node positive patients, difference in carboplatin dose and cyclophosphamide exposure in CALGB 40603. 


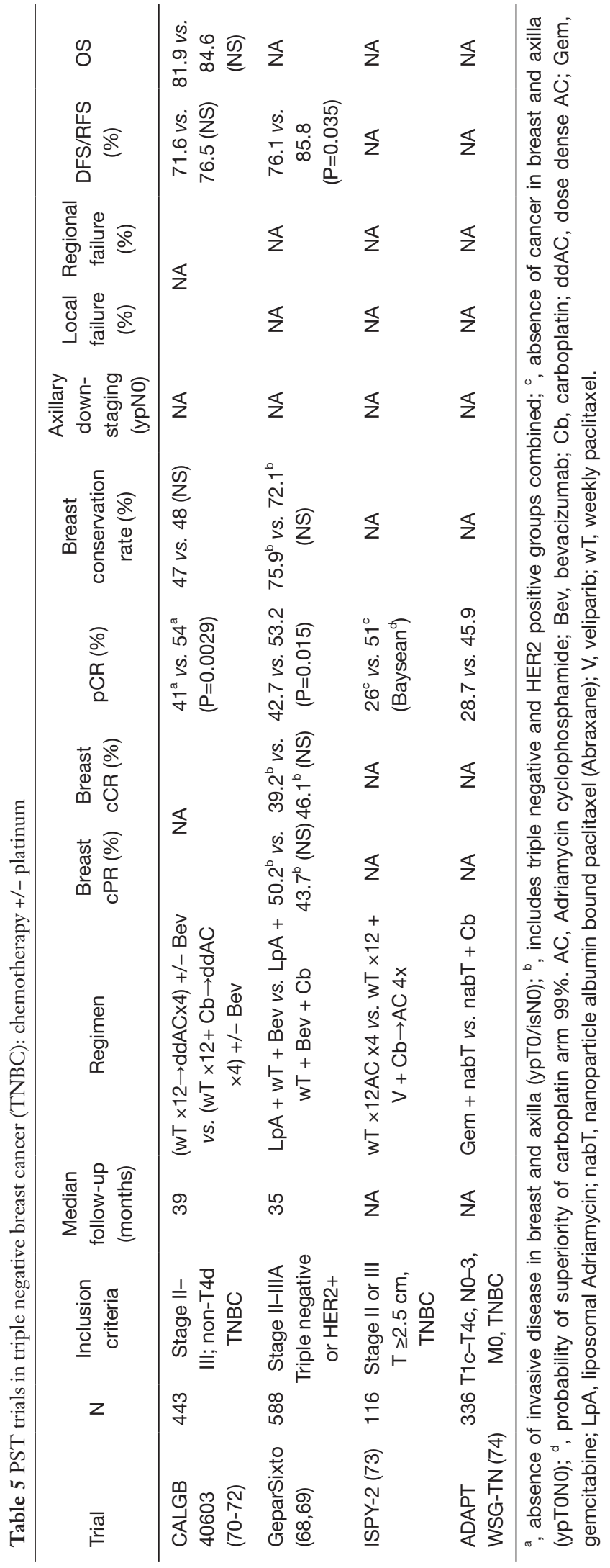

The impact of PST on breast conservation was explored in a surgical companion study to CALGB 40603 (71). In this study, $42 \%$ of patients were not eligible for breast conserving surgery at baseline. After PST, the proportion of patients eligible for breast conservation increased from 54\% to $68 \%$. Conversion from breast conservation ineligible to eligible was consistent with $\mathrm{pCR}$, such that conversion rates were numerically higher among patients who received carboplatin or bevacizumab or both in addition to standard PST compared to patients who received standard PST alone. Despite conversion to breast conservation eligibility, $31 \%$ of these patients chose to have mastectomy, as such the rates of breast conserving surgery was the same in patients randomized to carboplatin or not, despite higher pCR rates with the former. These findings are consistent with the results of a recent meta-analysis which failed to find an association between pCR and breast conservation rate (75).

\section{Neoadjuvant endocrine therapy}

The majority of preoperative endocrine therapy trials has been conducted in postmenopausal women with hormone receptor positive breast cancer (76-80) and is summarized in Table 6.

pCR rates are low with preoperative endocrine therapy and these trials have focused instead on other endpoints such as clinical response, breast conservation rates and Ki67 index, an immunohistochemical measure of cellular proliferation which has been found to correlate with treatment efficacy and prognosis. In the IMPACT trial comparing preoperative anastrozole, tamoxifen or the combination in postmenopausal women with hormone receptor positive breast cancer, greater geometric mean suppression of Ki67 was seen with anastrozole compared with tamoxifen or the combination. Higher Ki67 level after 2 weeks of preoperative endocrine therapy was associated with lower recurrence free survival $(81,82)$.

In a meta-analysis of 4 randomized trials (IMPACT, P024, PROACT and Exemestane versus Tamoxifen), preoperative aromatase inhibitors were found to be more effective than tamoxifen in terms of clinical objective response $(\mathrm{RR}=1.29 ; 95 \% \mathrm{CI}: 1.14-1.47 ; \mathrm{P}<0.001)$, ultrasound objective response $(\mathrm{RR}=1.29 ; 95 \% \mathrm{CI}$ : $1.10-1.51 ; \mathrm{P}=0.002)$ and breast conserving surgery rate $(\mathrm{RR}=1.36 ; 95 \% \mathrm{CI}: 1.16-1.59 ; \mathrm{P}<0.001)$ (83). The efficacy of preoperative aromatase inhibitors was further confirmed in the ACOSOG Z1031 randomized phase II trial comparing anastrozole, letrozole and exemestane in a 


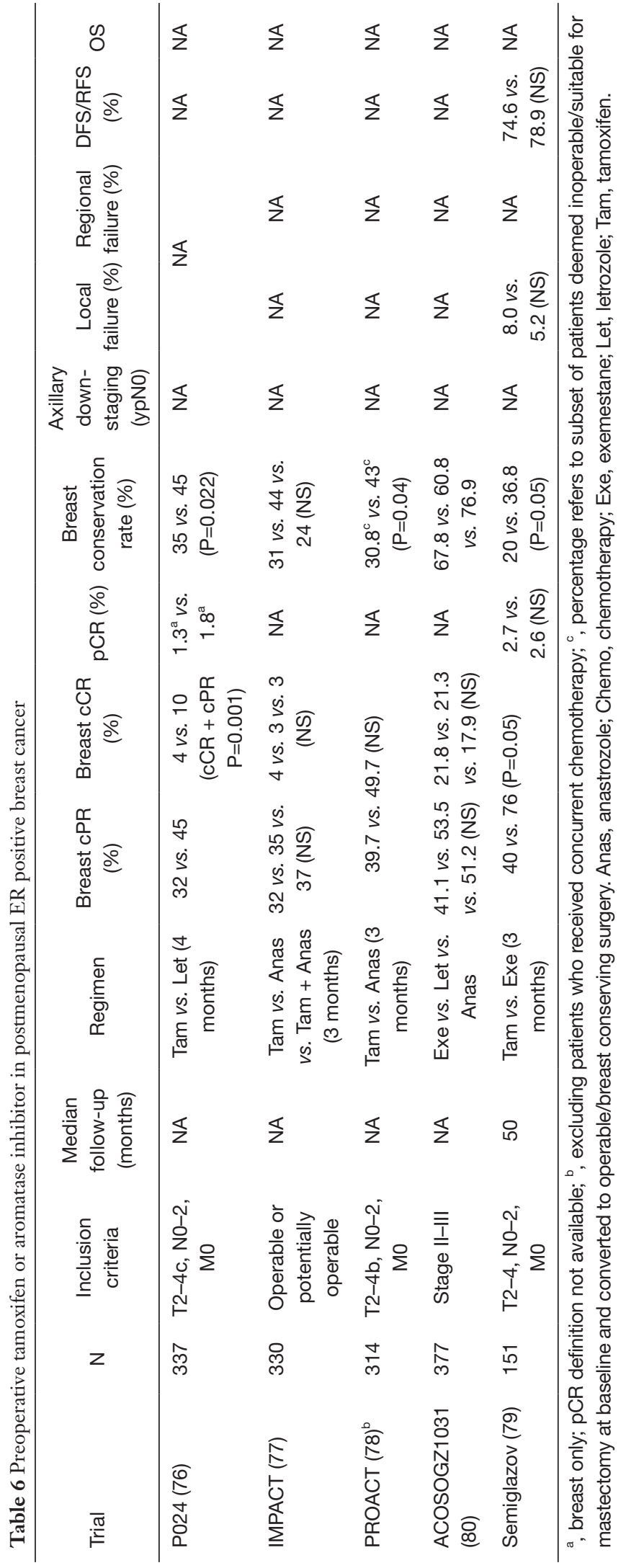

cohort of patients deemed to be of borderline suitability for breast conserving surgery or suitable only for mastectomy (79). All 3 drugs showed similar high rates of objective response $(62.9-74.8 \%)$ and breast conservation rate (60.8$76.9 \%)$.

In terms of optimal duration of preoperative endocrine therapy, a retrospective study showed that prolonging treatment beyond 3 months increased objective clinical response from $69.8 \%$ to $83.5 \%$ and breast conservation rate from $60 \%$ to $80 \%$ (84). Based on this, international guidelines recommend that the duration of preoperative endocrine therapy should be at least 3-4 months (85).

\section{Summary and conclusions}

PST has been shown to achieve high clinical response rates ranging from $49 \%$ to $82 \%$ in first generation trials. Although PST does not improve disease free or overall survival compared to AST, the rate of breast conserving surgery and proportion of patients with negative axillary nodes are increased with PST compared to upfront surgery. Conversely, the risk of local recurrence is increased with PST when surgery is omitted in patients with good clinical response or when patients are converted from planned mastectomy to breast conserving surgery after PST, although these data were derived in the era before the availability of taxanes and targeted therapy. Careful preoperative evaluation and assessment of surgical margins in patients who have received PST may mitigate this risk.

The pCR is an important surrogate endpoint for improved disease free and overall survival, except in low grade hormone receptor positive breast cancer. Although multiple PST trials have found that patients with pCR have improved survival outcomes compared to those with non pCR, it has been difficult to demonstrate that drugs which increase pCR rate lead to improvements in survival outcomes compared to the control drug. It is postulated that large sample sizes and marked increments in pCR are needed to demonstrate such a benefit.

The addition of taxanes to anthracyclines increases pCR rates and reduces the risk of local recurrence. In the PST setting, taxanes should be administered sequentially rather than concurrently with anthracyclines and weekly paclitaxel is superior to 3 weekly paclitaxel. Adapting PST to treatment response may be associated with improved survival outcomes, and the use of capecitabine as adjuvant therapy improves disease free and overall survival in patients with HER2 negative breast cancer who do not achieve pCR 
after anthracycline and taxane based PST.

Despite the dramatic improvements in pCR rates seen with the advent of taxanes, single and dual antiHER2 targeted therapy in HER2 positive breast cancer and platinums in triple negative breast cancer, there has not been a corresponding increment in the rate of breast conservation. This has been attributed to various factors such as tumour location, multicentricity, inability to predict pCR during preoperative assessment as well as physician and/or patient perception and preference.

Preoperative endocrine therapy is a feasible option in postmenopausal hormone receptor positive breast cancer. Aromatase inhibitors are superior too tamoxifen and high clinical response rates and breast conservation rates can be achieved with at least 3 to 4 months of therapy.

\section{Acknowledgements}

None.

\section{Footnote}

Conflicts of Interest: The author has no conflicts of interest to declare.

\section{References}

1. Kaufmann M, von Minckwitz G, Smith R, et al. International expert panel on the use of primary (preoperative) systemic treatment of operable breast cancer: review and recommendations. J Clin Oncol 2003;21:2600-8.

2. Fisher B, Brown A, Mamounas E, et al. Effect of preoperative chemotherapy on local-regional disease in women with operable breast cancer: findings from National Surgical Adjuvant Breast and Bowel Project B-18. J Clin Oncol 1997;15:2483-93.

3. Fisher B, Bryant J, Wolmark N, et al. Effect of preoperative chemotherapy on the outcome of women with operable breast cancer. J Clin Oncol 1998;16:2672-85.

4. Wolmark N, Wang J, Mamounas E, et al. Preoperative chemotherapy in patients with operable breast cancer: nine-year results from National Surgical Adjuvant Breast and Bowel Project B-18. J Natl Cancer Inst Monogr 2001;30:96-102.

5. Gianni L, Baselga J, Eiermann W, et al. Feasibility and tolerability of sequential doxorubicin/paclitaxel followed by cyclophosphamide, methotrexate, and fluorouracil and its effects on tumor response as preoperative therapy. Clin Cancer Res 2005;11:8715-21.

6. Gianni L, Baselga J, Eiermann W, et al. Phase III trial evaluating the addition of paclitaxel to doxorubicin followed by cyclophosphamide, methotrexate, and fluorouracil, as adjuvant or primary systemic therapy: European Cooperative Trial in Operable Breast Cancer. J Clin Oncol 2009;27:2474-81.

7. van der Hage JA, van de Velde CJ, Julien JP, et al. Preoperative chemotherapy in primary operable breast cancer: results from the European Organization for Research and Treatment of Cancer trial 10902. J Clin Oncol 2001;19:4224-37.

8. van Nes JG, Putter H, Julien JP, et al. Preoperative chemotherapy is safe in early breast cancer, even after 10 years of follow-up; clinical and translational results from the EORTC trial 10902. Breast Cancer Res Treat 2009;115:101-13.

9. Taucher S, Steger GG, Jakesz R, et al. The potential risk of neoadjuvant chemotherapy in breast cancer patientsresults from a prospective randomized trial of the Austrian Breast and Colorectal Cancer Study Group (ABCSG-07). Breast Cancer Res Treat 2008;112:309-16.

10. Scholl SM, Fourquet A, Asselain B, et al. Neoadjuvant versus adjuvant chemotherapy in premenopausal patients with tumours considered too large for breast conserving surgery: preliminary results of a randomised trial: S6. Eur J Cancer 1994;30A:645-52.

11. Mauri D, Pavlidis N, Ioannidis JP. Neoadjuvant versus adjuvant systemic treatment in breast cancer: a metaanalysis. J Natl Cancer Inst 2005;97:188-94.

12. Early Breast Cancer Trialists' Collaborative Group (EBCTCG). Long-term outcomes for neoadjuvant versus adjuvant chemotherapy in early breast cancer: metaanalysis of individual patient data from ten randomised trials. Lancet Oncol 2018;19:27-39.

13. Ketcham AS, Kinsey DL, Wexler H, et al. The development of spontaneous metastases after the removal of a "primary" tumor. II. Standardization protocol of 5 animal tumors. Cancer 1961;14:875-82.

14. Fisher B, Gunduz N, Saffer EA. Influence of the interval between primary tumor removal and chemotherapy on kinetics and growth of metastases. Cancer Res 1983;43:1488-92.

15. De Lena M, Zucali R, Viganotti G, et al. Combined chemotherapy-radiotherapy approach in locally advanced (T3b-T4) breast cancer. Cancer Chemother Pharmacol 1978;1:53-9. 
16. Swain SM, Sorace RA, Bagley CS, et al. Neoadjuvant chemotherapy in the combined modality approach of locally advanced nonmetastatic breast cancer. Cancer Res 1987;47:3889-94.

17. Bonadonna G, Veronesi U, Brambilla C, et al. Primary chemotherapy to avoid mastectomy in tumors with diameters of three centimeters or more. J Natl Cancer Inst 1990;82:1539-45.

18. Smith IE, Walsh G, Jones A, et al. High complete remission rates with primary neoadjuvant infusional chemotherapy for large early breast cancer. J Clin Oncol 1995;13:424-9.

19. Cochrane RA, Valasiadou P, Wilson AR, et al. Cosmesis and satisfaction after breast-conserving surgery correlates with the percentage of breast volume excised. Br J Surg 2003;90:1505-9.

20. Curigliano G, Burstein HJ, P Winer E, et al. De-escalating and escalating treatments for early-stage breast cancer: the St. Gallen International Expert Consensus Conference on the Primary Therapy of Early Breast Cancer 2017. Ann Oncol 2017;28:1700-12.

21. Boughey JC, Suman VJ, Mittendorf EA, et al. Sentinel lymph node surgery after neoadjuvant chemotherapy in patients with node-positive breast cancer: the ACOSOG Z1071 (Alliance) clinical trial. JAMA 2013;310:1455-61.

22. Kuehn T, Bauerfeind I, Fehm T, et al. Sentinel-lymphnode biopsy in patients with breast cancer before and after neoadjuvant chemotherapy (SENTINA): a prospective, multicentre cohort study. Lancet Oncol 2013;14:609-18.

23. Forrest AP, Levack PA, Chetty U, et al. A human tumour model. Lancet 1986;2:840-2.

24. Masuda N, Lee SJ, Ohtani S, et al. Adjuvant Capecitabine for Breast Cancer after Preoperative Chemotherapy. N Engl J Med 2017;376:2147-59.

25. Bear HD, Anderson S, Smith RE, et al. Sequential preoperative or postoperative docetaxel added to preoperative doxorubicin plus cyclophosphamide for operable breast cancer: National Surgical Adjuvant Breast and Bowel Project Protocol B-27. J Clin Oncol 2006;24:2019-27.

26. Kuerer HM, Newman LA, Smith TL, et al. Clinical course of breast cancer patients with complete pathologic primary tumor and axillary lymph node response to doxorubicin-based neoadjuvant chemotherapy. J Clin Oncol 1999; 17:460-9.

27. Cortazar P, Zhang L, Untch M, et al. Pathological complete response and long-term clinical benefit in breast cancer: the CTNeoBC pooled analysis. Lancet
2014;384:164-72.

28. von Minckwitz G, Untch M, Blohmer JU. et al. Definition and Impact of Pathologic Complete Response on Prognosis After Neoadjuvant Chemotherapy in Various Intrinsic Breast Cancer Subtypes. J Clin Oncol 2012;30:1796-804.

29. Office of Communications, Division of Drug Information, Center for Drug Evaluation and research, Food and Drug Administration. Guidance for industry: pathological complete response in neoadjuvant treatment of high-risk early-stage breast cancer: Use as an endpoint to support accelerated approval. October 2014. Available online: http://www.fda.gov/downloads/Drugs/ceComplianceRegu latoryInformation/Guidances/UCM305501.pdf

30. Mauriac L, MacGrogan G, Avril A, et al. Neoadjuvant chemotherapy for operable breast carcinoma larger than $3 \mathrm{~cm}$ : a unicentre randomized trial with a 124-month median follow-up. Institut Bergonié Bordeaux Groupe Sein (IBBGS). Ann Oncol 1999;10:47-52.

31. Bear HD, Anderson S, Brown A, et al. The effect on tumor response of adding sequential preoperative docetaxel to preoperative doxorubicin and cyclophosphamide: preliminary results from National Surgical Adjuvant Breast and Bowel Project Protocol B-27. J Clin Oncol 2003;21:4165-74.

32. Smith IC, Heys SD, Hutcheon AW, et al. Neoadjuvant chemotherapy in breast cancer: significantly enhanced response with docetaxel. J Clin Oncol 2002;20:1456-66.

33. Heys SD, Hutcheon AW, Sarkar TK, et al. Neoadjuvant docetaxel in breast cancer: 3-year survival results from the Aberdeen trial. Clin Breast Cancer 2002;3 Suppl 2:S69-74.

34. Hutcheon AW, Heys SD, Sarkar TK, et al. Neoadjuvant docetaxel in locally advanced breast cancer. Breast Cancer Res Treat 2003;79 Suppl 1:S19-24.

35. Diéras V, Fumoleau P, Romieu G, et al. Randomized parallel study of doxorubicin plus paclitaxel and doxorubicin plus cyclophosphamide as neoadjuvant treatment of patients with breast cancer. J Clin Oncol 2004;22:4958-65.

36. Evans TR, Yellowlees A, Foster E, et al. Phase III randomized trial of doxorubicin and docetaxel versus doxorubicin and cyclophosphamide as primary medical therapy in women with breast cancer: an angloceltic cooperative oncology group study. J Clin Oncol 2005;23:2988-95.

37. von Minckwitz G, Raab G, Caputo A, et al. Doxorubicin with cyclophosphamide followed by docetaxel every 21 days compared with doxorubicin and docetaxel every 14 
days as preoperative treatment in operable breast cancer: the GEPARDUO study of the German Breast Group. J Clin Oncol 2005;23:2676-85.

38. Kaufmann M, Eiermann W, Schuette M, et al. Longterm results from the neoadjuvant GeparDuo trial: A randomized, multicenter, open phase III study comparing a dose-intensified 8-week schedule of doxorubicin hydrochloride and docetaxel (ADoc) with a sequential 24-week schedule of doxorubicin hydrochloride/ cyclophosphamide followed by docetaxel (AC-Doc) regimen as preoperative therapy (NACT) in patients (pts) with operable breast cancer (BC). J Clin Oncol 2010;28:537.

39. Untch M, Möbus V, Kuhn W, et al. Intensive dose-dense compared with conventionally scheduled preoperative chemotherapy for high-risk primary breast cancer. J Clin Oncol 2009;27:2938-45.

40. Untch M, von Minckwitz G, Konecny GE, et al. PREPARE trial: a randomized phase III trial comparing preoperative, dose-dense, dose-intensified chemotherapy with epirubicin, paclitaxel, and CMF versus a standarddosed epirubicin-cyclophosphamide followed by paclitaxel with or without darbepoetin alfa in primary breast cancer-outcome on prognosis. Ann Oncol 2011;22:1999-2006.

41. Untch M, Fasching PA, Konecny GE, et al. PREPARE trial: a randomized phase III trial comparing preoperative, dose-dense, dose-intensified chemotherapy with epirubicin, paclitaxel and CMF versus a standard-dosed epirubicin/ cyclophosphamide followed by paclitaxel \pm darbepoetin alfa in primary breast cancer-results at the time of surgery. Ann Oncol 2011;22:1988-98.

42. Therasse P, Mauriac L, Welnicka-Jaskiewicz M, et al. Final results of a randomized phase III trial comparing cyclophosphamide, epirubicin, and fluorouracil with a dose-intensified epirubicin and cyclophosphamide + filgrastim as neoadjuvant treatment in locally advanced breast cancer: an EORTC-NCIC-SAKK multicenter study. J Clin Oncol 2003;21:843-50.

43. Steger GG, Galid A, Gnant M, et al. Pathologic complete response with six compared with three cycles of neoadjuvant epirubicin plus docetaxel and granulocyte colony-stimulating factor in operable breast cancer: results of ABCSG-14. J Clin Oncol 2007;25:2012-8.

44. Green MC, Buzdar AU, Smith T, et al. Weekly paclitaxel improves pathologic complete remission in operable breast cancer when compared with paclitaxel once every 3 weeks. J Clin Oncol 2005;23:5983-92.

45. von Minckwitz G, Kümmel S, Vogel P. Neoadjuvant vinorelbine-capecitabine versus docetaxel-doxorubicincyclophosphamide in early nonresponsive breast cancer: phase III randomized GeparTrio trial. J Natl Cancer Inst 2008;100:542-51.

46. von Minckwitz G, Kümmel S, Vogel P, et al. Intensified neoadjuvant chemotherapy in early-responding breast cancer: phase III randomized GeparTrio study. J Natl Cancer Inst 2008;100:552-62.

47. von Minckwitz G, Blohmer JU, Costa SD, et al. Responseguided neoadjuvant chemotherapy for breast cancer. J Clin Oncol 2013;31:3623-30.

48. Slamon DJ, Clark GM, Wong SG, et al. Human breast cancer: correlation of relapse and survival with amplification of the HER-2/neu oncogene. Science 1987;235:177-82.

49. Gianni L, Eiermann W, Semiglazov V, et al. Neoadjuvant chemotherapy with trastuzumab followed by adjuvant trastuzumab versus neoadjuvant chemotherapy alone, in patients with HER2-positive locally advanced breast cancer (the NOAH trial): a randomised controlled superiority trial with a parallel HER2-negative cohort. Lancet 2010;375:377-84.

50. Gianni L, Eiermann W, Semiglazov V, et al. Neoadjuvant and adjuvant trastuzumab in patients with HER2-positive locally advanced breast cancer (NOAH): follow-up of a randomised controlled superiority trial with a parallel HER2-negative cohort. Lancet Oncol 2014;15:640-7.

51. Buzdar AU, Ibrahim NK, Francis D, et al. Significantly higher pathologic complete remission rate after neoadjuvant therapy with trastuzumab, paclitaxel, and epirubicin chemotherapy: results of a randomized trial in human epidermal growth factor receptor 2-positive operable breast cancer. J Clin Oncol 2005;23:3676-85.

52. Buzdar AU, Valero V, Ibrahim NK, et al. Neoadjuvant therapy with paclitaxel followed by 5 -fluorouracil, epirubicin, and cyclophosphamide chemotherapy and concurrent trastuzumab in human epidermal growth factor receptor 2-positive operable breast cancer: an update of the initial randomized study population and data of additional patients treated with the same regimen. Clin Cancer Res 2007;13:228-33.

53. Untch M, Rezai M, Loibl S, et al. Neoadjuvant treatment with trastuzumab in HER2-positive breast cancer: results from the GeparQuattro study. J Clin Oncol 2010;28:2024-31.

54. Untch M, Fasching PA, Konecny GE, et al. Pathologic complete response after neoadjuvant chemotherapy plus trastuzumab predicts favorable survival in human 
epidermal growth factor receptor 2-overexpressing breast cancer: results from the TECHNO trial of the AGO and GBG study groups. J Clin Oncol 2011;29:3351-7.

55. Untch M, Loibl S, Bischoff J, et al. Lapatinib versus trastuzumab in combination with neoadjuvant anthracycline-taxane-based chemotherapy (GeparQuinto, GBG 44): a randomised phase 3 trial. Lancet Oncol 2012;13:135-44.

56. Guarneri V, Frassoldati A, Bottini A, et al. Preoperative chemotherapy plus trastuzumab, lapatinib, or both in human epidermal growth factor receptor 2-positive operable breast cancer: results of the randomized phase II CHER-LOB study. J Clin Oncol 2012;30:1989-95.

57. Baselga J, Bradbury I, Eidtmann H, et al. Lapatinib with trastuzumab for HER2-positive early breast cancer (NeoALTTO): a randomised, open-label, multicentre, phase 3 trial. Lancet 2012;379:633-40.

58. de Azambuja E, Holmes AP, Piccart-Gebhart M, et al. Lapatinib with trastuzumab for HER2-positive early breast cancer (NeoALTTO): survival outcomes of a randomised, open-label, multicentre, phase 3 trial and their association with pathological complete response. Lancet Oncol 2014;15:1137-46.

59. Carey LA, Berry DA, Cirrincione CT, et al. Molecular Heterogeneity and Response to Neoadjuvant Human Epidermal Growth Factor Receptor 2 Targeting in CALGB 40601, a Randomized Phase III Trial of Paclitaxel Plus Trastuzumab With or Without Lapatinib. J Clin Oncol 2016;34:542-9.

60. Golshan M, Cirrincione CT, Sikov WM, et al. Impact of neoadjuvant therapy on eligibility for and frequency of breast conservation in stage II-III HER2-positive breast cancer: surgical results of CALGB 40601 (Alliance). Breast Cancer Res Treat 2016;160:297-304.

61. Robidoux A, Tang G, Rastogi P, et al. Lapatinib as a component of neoadjuvant therapy for HER2-positive operable breast cancer (NSABP protocol B-41): an open-label, randomised phase 3 trial. Lancet Oncol 2013;14:1183-92.

62. Gianni L, Pienkowski T, Im YH, et al. Efficacy and safety of neoadjuvant pertuzumab and trastuzumab in women with locally advanced, inflammatory, or early HER2-positive breast cancer (NeoSphere): a randomised multicentre, open-label, phase 2 trial. Lancet Oncol 2012;13:25-32.

63. Gianni L, Pienkowski T, Im YH, et al. 5-year analysis of neoadjuvant pertuzumab and trastuzumab in patients with locally advanced, inflammatory, or early-stage
HER2-positive breast cancer (NeoSphere): a multicentre, open-label, phase 2 randomised trial. Lancet Oncol 2016;17:791-800.

64. Schneeweiss A, Chia S, Hickish T, et al. Pertuzumab plus trastuzumab in combination with standard neoadjuvant anthracycline-containing and anthracycline-free chemotherapy regimens in patients with HER2-positive early breast cancer: a randomized phase II cardiac safety study (TRYPHAENA). Ann Oncol 2013;24:2278-84.

65. Schneeweiss A, Chia S, Hickish T, et al. Long-term efficacy analysis of the randomised, phase II TRYPHAENA cardiac safety study: Evaluating pertuzumab and trastuzumab plus standard neoadjuvant anthracycline-containing and anthracycline-free chemotherapy regimens in patients with HER2-positive early breast cancer. Eur J Cancer 2018;89:27-35.

66. Carey L, Winer E, Viale G, et al. Triple-negative breast cancer: disease entity or title of convenience? Nat Rev Clin Oncol 2010;7:683-92.

67. Turner N, Tutt A, Ashworth A. Hallmarks of 'BRCAness' in sporadic cancers. Nat Rev Cancer 2004;4:814-9.

68. von Minckwitz G, Schneeweiss A, Loibl S, et al. Neoadjuvant carboplatin in patients with triple-negative and HER2-positive early breast cancer (GeparSixto; GBG 66): a randomised phase 2 trial. Lancet Oncol 2014;15:747-56.

69. Untch M, Schneeweiss A, Salat C, et al. 163PD Longterm survival analysis of the randomized phase II trial investigating the addition of carboplatin to neoadjuvant therapy for triple-negative (TNBC) and HER2positive early breast cancer (GeparSixto). Ann Oncol 2017;28:v43-67.

70. Sikov WM, Berry DA, Perou CM, et al. Impact of the addition of carboplatin and/or bevacizumab to neoadjuvant once-per-week paclitaxel followed by dosedense doxorubicin and cyclophosphamide on pathologic complete response rates in stage II to III triple-negative breast cancer: CALGB 40603 (Alliance). J Clin Oncol 2015;33:13-21.

71. Golshan M, Cirrincione CT, Sikov WM, et al. Impact of neoadjuvant chemotherapy in stage II-III triple negative breast cancer on eligibility for breast-conserving surgery and breast conservation rates: surgical results from CALGB 40603 (Alliance). Ann Surg 2015;262:434-9; discussion 438-9.

72. Sikov WM, Berry DA, Perou CM, et al. Event-free and overall survival following neoadjuvant weekly paclitaxel and dose-dense $\mathrm{AC}+$ /- carboplatin and/or bevacizumab 
in triple-negative breast cancer: Outcomes from CALGB 40603 (Alliance). Cancer Res 2016;76:Abstract nr S2-05.

73. Rugo HS, Olopade OI, DeMichele A, et al. Adaptive Randomization of Veliparib-Carboplatin Treatment in Breast Cancer. N Engl J Med 2016;375:23-34.

74. Gluz O, Nitz U, Liedtke C, et al. Comparison of Neoadjuvant Nab-Paclitaxel+Carboplatin vs NabPaclitaxel+Gemcitabine in Triple-Negative Breast Cancer: Randomized WSG-ADAPT-TN Trial Results. J Natl Cancer Inst 2018;110:628-37.

75. Criscitiello C, Golshan M, Barry WT, et al. Impact of neoadjuvant chemotherapy and pathological complete response on eligibility for breast-conserving surgery in patients with early breast cancer: A meta-analysis. Eur J Cancer 2018;97:1-6.

76. Eiermann W, Paepke S, Appfelstaedt J, et al. Preoperative treatment of postmenopausal breast cancer patients with letrozole: A randomized double-blind multicenter study. Ann Oncol 2001;12:1527-32.

77. Smith IE, Dowsett M, Ebbs SR, et al. Neoadjuvant treatment of postmenopausal breast cancer with anastrozole, tamoxifen, or both in combination: the Immediate Preoperative Anastrozole, Tamoxifen, or Combined with Tamoxifen (IMPACT) multicenter doubleblind randomized trial. J Clin Oncol 2005;23:5108-16.

78. Cataliotti L, Buzdar AU, Noguchi S, et al. Comparison of anastrozole versus tamoxifen as preoperative therapy in postmenopausal women with hormone receptor-positive breast cancer: the Pre-Operative "Arimidex" Compared to Tamoxifen (PROACT) trial. Cancer 2006;106:2095-103.

79. Semiglazov V, Kletsel A, Semiglazov V et al. Exemestane
(E) vs tamoxifen (T) as neoadjuvant endocrine therapy for postmenopausal women with ER+ breast cancer (T2N1-2, T3N0-1, T4N0M0). J Clin Oncol 2005;23:530.

80. Ellis MJ, Suman VJ, Hoog J, et al. Randomized phase II neoadjuvant comparison between letrozole, anastrozole, and exemestane for postmenopausal women with estrogen receptor-rich stage 2 to 3 breast cancer: clinical and biomarker outcomes and predictive value of the baseline PAM50-based intrinsic subtype--ACOSOG Z1031. J Clin Oncol 2011;29:2342-9.

81. Dowsett M, Ebbs SR, Dixon JM, et al. Biomarker changes during neoadjuvant anastrozole, tamoxifen, or the combination: influence of hormonal status and HER-2 in breast cancer--a study from the IMPACT trialists. J Clin Oncol 2005;23:2477-92.

82. Dowsett M, Smith IE, Ebbs SR, et al. Prognostic value of Ki67 expression after short-term presurgical endocrine therapy for primary breast cancer. J Natl Cancer Inst 2007;99:167-70.

83. Seo JH, Kim YH, Kim JS. Meta-analysis of pre-operative aromatase inhibitor versus tamoxifen inpostmenopausal woman with hormone receptor-positive breast cancer. Cancer Chemother Pharmacol 2009;63:261-6.

84. Dixon JM, Renshaw L, Macaskill EJ, et al. Increase in response rate by prolonged treatment with neoadjuvant letrozole. Breast Cancer Res Treat 2009;113:145-51.

85. Kaufmann M, von Minckwitz G, Mamounas EP, et al. Recommendations from an international consensus conference on the current status and future of neoadjuvant systemic therapy in primary breast cancer. Ann Surg Oncol 2012;19:1508-16.
Cite this article as: Wong NS. Primary medical therapy and breast conservation treatment: the medical oncology perspective. Gland Surg 2018;7(6):560-575. doi: 10.21037/gs.2018.10.02 